\title{
SPLIT BRAIDS
}

\author{
STEPHEN P. HUMPHRIES
}

(Communicated by Warren J. Wong)

\begin{abstract}
Let $B_{n}$ be the group of braids on $n$ strings with standard generators $\sigma_{1}, \ldots, \sigma_{n-1}$. For $i \in\{1,2, \ldots, n-1\}$ we let $B_{n}^{i}$ be the subgroup of $B_{n}$ generated by the elements $\sigma_{1}, \ldots, \sigma_{i-1}, \sigma_{i+1}, \ldots, \sigma_{n-1}$. In this paper we give an algorithm for deciding if, given $\alpha \in B_{n}$ there is $i \in$ $\{1,2, \ldots, n-1\}$ such that $\alpha$ is conjugate into $B_{n}^{i}$. We call such a braid a split braid. Such a split braid gives rise to a split link. This algorithm gives a partial solution to the problem of finding braids that represent reducible mapping classes. It also represents a contribution to the algebraic link problem and it gives a way of determining if a braid in $B_{n}$ can be conjugated into the subgroup $B_{n-1}$, which we identify with $B_{n-1}^{n-1}$.
\end{abstract}

\section{INTRODUCTION}

For $n>1$ let $B_{n}$ be the group of braids on $n$ strings. Then $B_{n}$ has a presentation as a group with generators $\sigma_{1}, \ldots, \sigma_{n-1}$ and relations

$$
\begin{aligned}
& \sigma_{i} \sigma_{j}=\sigma_{j} \sigma_{i} \quad \text { if } 1 \leq i, j \leq n-1 \text { and }|i-j|>1 ; \\
& \sigma_{i} \sigma_{i+1} \sigma_{i}=\sigma_{i+1} \sigma_{i} \sigma_{i+1} \quad \text { for } 1 \leq i<n-1 .
\end{aligned}
$$

It is also well known that $B_{n}$ has a faithful representation in $\operatorname{Aut}(F(n))$, the group of automorphisms of the free group $F(n)$ of rank $n$. If $x_{1}, \ldots, x_{n}$ are fixed free generators for $F(n)$, then the action of $B_{n}$ on $F(n)$ is given by the following actions of the generators $\sigma_{1}, \ldots, \sigma_{n-1}$ of $B_{n}$ on the generators $x_{1}, \ldots, x_{n}$ of $F(n)$ :

$$
\sigma_{i}\left(x_{j}\right)= \begin{cases}x_{j} & \text { if } j \neq i, i+1, \\ x_{i+1} & \text { if } j=i, \\ x_{i+1}^{-1} x_{i} x_{i+1} & \text { if } j=i+1 .\end{cases}
$$

It is easy to check that with this action the word $x_{1} x_{2} \cdots x_{n}$ is fixed and that if $\alpha \in B_{n}$, then each $\alpha\left(x_{i}\right)$ is a conjugate of some $x_{j}$. In fact these two properties characterize the image of or braid group in $\operatorname{Aut}(F(n))$ and we

Received by the editors March 13, 1990 and, in revised form, August 10, 1990.

1980 Mathematics Subject Classification (1985 Revision). Primary 20F36; Secondary 57M25.

Key words and phrases. Braid group, algorithm. 
sometimes confuse a braid $\alpha$ with the $n$-tuple of words $\left(\alpha\left(x_{1}\right), \ldots, \alpha\left(x_{n}\right)\right)$. This representation of the braid groups has a geometric interpretation since $B_{n}$ acts as a group of mapping classes of an $n$-punctured disc $D_{n}$. The above action can then be realized as the action of $B_{n}$ on the fundamental group of $D_{n}$, which is a free group of rank $n$. We identify this group with $F(n)$. Let $\pi_{n}: B_{n} \rightarrow S_{n}$ be the permutation representation of $B_{n}$ onto the symmetric group. For more information on braid groups see [Bi, Ma].

For $i \in\{1,2, \ldots, n-1\}$ we let $B_{n}^{i}$ be the subgroup of $B_{n}$ generated by the elements $\sigma_{1}, \ldots, \sigma_{i-1}, \sigma_{i+1}, \ldots, \sigma_{n-1}$. In this paper we give an algorithm for determining if, given $\alpha \in B_{n}$, there is $i \in\{1,2, \ldots, n-1\}$ such that $\alpha$ is conjugate into $B_{n}^{i}$. We call such a braid a split braid. This algorithm gives a partial solution to the problem of finding braids that represent reducible mapping classes, i.e. braids whose action on the disc $D_{n}$ fixes the isotopy class of a nontrivial, nonboundary parallel simple closed curve on $D_{i n}$. Furthermore the closed braid $\hat{\alpha}$ corresponding to a split braid $\alpha$ is a split link in the sense that there is an $S^{2}$ disjoint from $\hat{\alpha}$ separating the components of $\hat{\alpha}$. Thus our result also represents a contribution to the algebraic link problem. Also note that in the case $i=n-1$ our method gives a way of determining if a braid in $B_{n}$ can be conjugated into the subgroup $B_{n}^{i}$, which we can identify with $B_{n-1}$.

\section{THE ALGORITHM}

For each subset $I \subset\{1,2, \ldots, n\}$ there is a map $\varphi_{I}: B_{n} \rightarrow B_{n}$, which consists of taking a braid $\alpha$ in $B_{n}$ and for each $i \in I$ pulling out the string that ends up in the $i$ th position after doing $\alpha$, and putting it back in as a straight string to the right of all the other strings. We think of $\varphi_{I}(\alpha)$ as belonging to the subgroup $B_{n}$ consisting of braids fixing the last $|I|$ strings, where $|I|$ is the cardinality of the set $I$. This process involves a relabelling of the strings and is illustrated in the case $n=4, I=\{3\}$ in Figure 1 .

Note that the map $\varphi_{I}$ is not a homomorphism; however, we do have the following result:

Lemma 2.1. Let $I \subset\{1,2, \ldots, n\}$ and $\alpha, \beta \in B_{n}$. Then $\varphi_{I}(\alpha \beta)=$ $\varphi_{I}(\alpha) \varphi_{J}(\beta)$, where $J=\pi\left(\alpha^{-1}\right)(I)$.

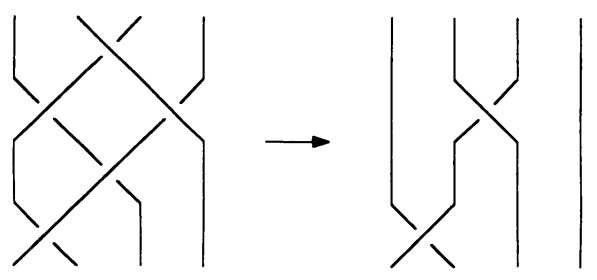

FIGURE 1 
Proof. The proof consists of noticing that if $i \in I$ and if $j=\pi\left(\alpha^{-1}\right)(i)$, then it is the $j$ th string of $\beta$ that is removed when we remove the $i$ th string of $\alpha$.

For $I \subset\{1,2, \ldots, n\}$ we let $Y_{I}$ be the stablizer of $I$ in $B_{n}$, i.e. the subgroup consisting of all the braids $\alpha$ such that $\pi\left(\alpha^{-1}\right)(I)=I$. Since $Y_{I}$ contains the pure braid group it follows easily that $Y_{I}$ is of finite index in $B_{n}$. Then the key observation is:

Proposition 2.2. Let $\alpha, \gamma \in Y_{I}$ and $\beta=\gamma \alpha \gamma^{-1}$. Then $\varphi_{I}(\beta)$ is a conjugate of $\alpha$ in $B_{n}$.

Proof. Let $\alpha, \gamma$, and $\beta$ be as above. Then Lemma 2.1 shows that

$$
\varphi_{I}(\beta)=\varphi_{I}\left(\gamma \alpha \gamma^{-1}\right)=\varphi_{I}(\gamma) \varphi_{I}(\alpha) \varphi_{I}\left(\gamma^{-1}\right),
$$

and so the result follows if we can show that $\varphi_{I}\left(\gamma^{-1}\right)=\varphi_{I}(\gamma)^{-1}$. Again Lemma 2.1 shows that we have, id $=\varphi_{I}\left(\gamma \gamma^{-1}\right)=\varphi_{I}(\gamma) \varphi_{I}\left(\gamma^{-1}\right)$ and so the result follows.

We next show that the definition of $\varphi_{I}$ amounts to the following algebraic operation as far as the braid automorphisms are concerned. If $m \leq n$, then we think of $F(m)$ as the subgroup of $F(n)$ generated by $x_{1}, \ldots, x_{m}$. We also use the notation $\alpha^{*}(I)$ instead of $\pi\left(\alpha^{-1}\right)(I)$. Note that we have

$$
(\alpha \beta)^{*}(I)=\pi\left((\alpha \beta)^{-1}\right)(I)=\pi\left(\beta^{-1}\right) \pi\left(\alpha^{-1}\right)(I)=\beta^{*} \alpha^{*}(I) .
$$

The next result is a consequence of Lemmas 2.4 and 2.5 .

Proposition 2.3. Suppose that $I \subset\{1,2, \ldots, n\}$. If $j \in\{1,2, \ldots, n\} \backslash I$ then we let $n(j)=n(j, I)$ be the number of elements of $I$ that are strictly less than $j$. If $\alpha \in B_{n}$, then $\varphi_{I}(\alpha)\left(x_{j}\right)=\Sigma_{I}\left(\alpha\left(x_{j}\right)\right)$ where $\Sigma_{I}: F(n) \rightarrow F(n-|I|)$ is the epimorphism defined by the following action on the generators of $F(n)$ :

$$
\begin{array}{ll}
\Sigma_{I}\left(x_{k}\right)=x_{k-n(k)} & \text { if } k \in\{1,2, \ldots, n\} \backslash I ; \\
\Sigma_{I}\left(x_{i}\right)=\text { identity } & \text { if } i \in I .
\end{array}
$$

We let $\Sigma_{I}$ act on the generators of $B_{n}$ in the following way:

$$
\begin{array}{cl}
\Sigma_{I}\left(\sigma_{k}\right)=\sigma_{k-n(k)} & \text { if } k \in\{1,2, \ldots, n-1\} \backslash I ; \\
\Sigma_{I}\left(\sigma_{i}\right)=\text { identity } & \text { if } i \in I .
\end{array}
$$

In what follows it is convenient to define $n(i)=\infty$ if $i \in I$ and to have the convention that $x_{-\infty}=$ id in $F(n), \sigma_{-\infty}=$ id in $B_{n}$.

Lemma 2.4. Let $X \in F(n)$ and $\varepsilon= \pm 1$.

(a) If $k, k+1 \in\{1,2, \ldots, n\} \backslash I$, then $\Sigma_{I}\left(\sigma_{k}^{\varepsilon}(X)\right)=\sigma_{k-n(k)}^{\varepsilon}\left(\Sigma_{I}(X)\right)$.

(b) If $k \in I$, but $k+1 \in\{1, \ldots, n\} \backslash I$, then $\Sigma_{I}\left(\sigma_{k}^{\varepsilon}(X)\right)=\Sigma_{I^{\prime}}(X)$ where $I^{\prime}=\sigma_{k}^{*}(I)$. 
(c) If $k$ does not belong to $I$ but $k+1$ does, then $\Sigma_{I}\left(\sigma_{k}^{\varepsilon}(X)\right)=\Sigma_{I^{\prime}}(X)$, where $I^{\prime}=\sigma_{k}^{*}(I)$.

(d) If $k, k+1 \in I$, then $\Sigma_{I}\left(\sigma_{k}^{\varepsilon}(X)\right)=\Sigma_{I}(X)$.

The functions $\Sigma_{I}$ satisfy

$$
\Sigma_{I}\left(\sigma_{k}^{\varepsilon} X\right)=\Sigma_{I \subset I^{\prime}}\left(\sigma_{k}^{\varepsilon}\right) \Sigma_{I^{\prime}}(X),
$$

where $I^{\prime}=\sigma_{k}^{*}(I)$ and $X \in F(n)$.

Proof. In each case below we only prove the result for $\varepsilon=+1$, the other case being similar. (a) Suppose that $k, k+1 \in\{1,2, \ldots, n\} \backslash I$. Then $n(k)=$ $n(k+1)$ and so we have

(i) $\Sigma_{I}\left(\sigma_{k}\left(x_{k}\right)\right)=\Sigma_{I}\left(x_{k+1}\right)=x_{k-n(k)+1}=\sigma_{k-n(k)}\left(x_{k-n(k)}\right)=\sigma_{k-n(k)}\left(\Sigma_{I}\left(x_{k}\right)\right)$;

(ii) $\Sigma_{I}\left(\sigma_{k}\left(x_{k+1}\right)\right)=\Sigma_{I}\left(x_{k+1}^{-1} x_{k} x_{k+1}\right)=x_{k+1-n(k)}^{-1} x_{k-n(k)} x_{k+1-n(k)}=$ $\sigma_{k-n(k)}\left(\Sigma_{I}\left(x_{k+1}\right)\right) ;$ and

(iii) If $j \neq k, k+1$, then $\Sigma_{I}\left(\sigma_{k}\left(x_{j}\right)\right)=\Sigma_{I}\left(x_{j}\right)=x_{j-n(j)}$ and $\sigma_{k-n(k)}\left(\Sigma_{I}\left(x_{j}\right)\right)=\sigma_{k-n(k)}\left(x_{j-n(j)}\right)$.

To see that $x_{j-n(j)}=\sigma_{k-n(k)}\left(x_{j-n(j)}\right)$ we consider case $1: j<k$, and case 2: $j>k+1$. In case 1 we have two subcases; (a) $j \in I$; and (b) $j$ not in $I$. If (a) then $x_{j-n(j)}=$ id and we are O.K. If (b), then $n(k)-n(j) \leq k-j-1<k-j$ showing that $k-n(k)>j-n(j)$, which gives the result. If we have case 2 , then again we consider the two cases (a) and (b) above. If we have (a), then $x_{j-n(j)}=\mathrm{id}$ and we are done. If (b), then $k, k+1$ not in $I$ means that $n(j)-n(k) \leq j-k-2$ and so $j-n(j)>k-n(k)+1$, which gives the result. This proves part (a) of Lemma 2.4 .

(b) Suppose that $k \in I$, but $k+1 \in\{1, \ldots, n\} \backslash I$. Let $I^{\prime}=\sigma_{k}^{*}(I)=$ $(I \backslash\{k\}) \cup\{k+1\}$. Then (i) $\Sigma_{I} \sigma_{k}\left(x_{k}\right)=\Sigma_{I}\left(x_{k+1}\right)=x_{k+1-n(k+1, I)}$ and $\Sigma_{I^{\prime}}\left(x_{k}\right)=$ $x_{k-n\left(k, I^{\prime}\right)}$; but it is easily seen that $n\left(k, I^{\prime}\right)=n(k+1, I)$, and so the result follows in this case. (ii) We have $\Sigma_{I} \sigma_{k}\left(x_{k+1}\right)=\Sigma_{I}\left(x_{k+1}^{-1} x_{k} x_{k+1}\right)=$ id and $\Sigma_{I^{\prime}}\left(x_{k+1}\right)=\mathrm{id}$. (iii) If $j \neq k, k+1$, then $\Sigma_{I} \sigma_{k}\left(x_{j}\right)=\Sigma_{I}\left(x_{j}\right)=x_{j-n(j, I)}$ and $\Sigma_{I^{\prime}}\left(x_{j}\right)=x_{j-n\left(j, I^{\prime}\right)}$. The result now follows from the fact that $n(j, I)=$ $n\left(j, I^{\prime}\right)$ if $j \neq k, k+1$.

(c) Suppose that $k+1 \in I$, but $k \in\{1, \ldots, n\} \backslash I$. Let $I^{\prime}=\sigma_{k}^{*}(I)=$ $(I \backslash\{k+1\}) \cup\{k\}$. Then (i) $\Sigma_{I} \sigma_{k}\left(x_{k}\right)=\Sigma_{I}\left(x_{k+1}\right)=$ id and $\Sigma_{I^{\prime}}\left(x_{k}\right)=$ id. (ii) $\Sigma_{I} \sigma_{k}\left(x_{k+1}\right)=\Sigma_{I}\left(x_{k+1}^{-1} x_{k} x_{k+1}\right)=x_{k-n(k, I)}$ and $\Sigma_{I^{\prime}}\left(x_{k+1}\right)=x_{k-n\left(k+1, I^{\prime}\right)}$; but it is easily seen that $n\left(k+1, I^{\prime}\right)=n(k, I)$ and so the result follows in this case. (iii) If $j \neq k, k+1$, then $\Sigma_{I} \sigma_{k}\left(x_{j}\right)=\Sigma_{I}\left(x_{j}\right)=x_{j-n(j, I)}$ and $\Sigma_{I^{\prime}}\left(x_{j}\right)=x_{j-n\left(j, I^{\prime}\right)}$. The result now follows since $n(j, I)=n\left(j, I^{\prime}\right)$ if $j \neq k, k+1$.

(d) If $k, k+1 \in I$, then $\Sigma_{I}\left(\sigma_{k}^{\varepsilon}\left(x_{j}\right)\right)=x_{j-n(j)}=\Sigma_{I}\left(x_{j}\right)$ for all $j$.

The remaining formula is easily checked using parts (a), (b), (c), and (d), which cover all cases.

In light of the above result we are now justified in writing $\Sigma_{I} \sigma_{k}^{\varepsilon} \alpha=$ $\Sigma_{I \cup I^{\prime}}\left(\sigma_{k}\right)^{\varepsilon} \Sigma_{I^{\prime}} \alpha$ where $\alpha \in B_{n}$. We do this in what follows. 
The idea for the proof of Proposition 2.3 is to show that $\Sigma_{I}$ has the same properties as does $\varphi_{I}$; so we next prove the analogue of Lemma 2.1:

Lemma 2.5. Let $\alpha, \beta \in B_{n}$. Then $\Sigma_{I}(\alpha \beta)=\Sigma_{I}(\alpha) \Sigma_{J}(\beta)$, where $J=\alpha^{*}(I)$.

Proof. We induct on the length $r$ of the word $\alpha$. Clearly the result is true in the case $r=0 \quad(\alpha=$ identity). So now suppose that the result is true for all words $\alpha$ of length $N \geq 0$ and suppose that $\alpha=\sigma_{k}^{\varepsilon} \alpha^{\prime}$, where $\varepsilon= \pm 1$ and the length of $\alpha^{\prime}$ is $N$. By induction we now have $\Sigma_{I}\left(\alpha^{\prime} \beta\right)=\Sigma_{I}\left(\alpha^{\prime}\right) \Sigma_{J}(\beta)$ for all $I \subset\{1, \ldots, n\}$, with $J=\left(\alpha^{\prime}\right)^{*}(I)$. Thus if $I \subset\{1, \ldots, n\}, I^{\prime}=\left(\sigma_{k}^{\varepsilon}\right)^{*}(I)$ and $J^{\prime}=\left(\alpha^{\prime}\right)^{*}\left(I^{\prime}\right)$, then by Lemma 2.4 we have

$$
\begin{aligned}
\Sigma_{I}(\alpha \beta) & =\Sigma_{I}\left(\sigma_{k}^{\varepsilon} \alpha^{\prime} \beta\right)=\Sigma_{I \cup I^{\prime}}\left(\sigma_{k}^{\varepsilon}\right) \Sigma_{I^{\prime}}\left(\alpha^{\prime} \beta\right) \\
& =\Sigma_{I \cup I^{\prime}}\left(\sigma_{k}^{\varepsilon}\right) \Sigma_{I^{\prime}}\left(\alpha^{\prime}\right) \Sigma_{J^{\prime}}(\beta) \\
& =\Sigma_{I}\left(\sigma_{k}^{\varepsilon} \alpha^{\prime}\right) \Sigma_{J^{\prime}}(\beta),
\end{aligned}
$$

which is what we require since

$$
J^{\prime}=\left(\alpha^{\prime}\right)^{*}\left(I^{\prime}\right)=\left(\alpha^{\prime}\right)^{*}\left(\left(\sigma_{k}^{\varepsilon}\right)^{*}(I)\right)=\left(\sigma_{k}^{\varepsilon} \alpha^{\prime}\right)^{*}(I) .
$$

Now Proposition 2.3 follows from the last result and the fact that $\varphi_{I} \sigma_{k}^{\varepsilon}=$ $\Sigma_{I} \sigma_{k}^{\varepsilon}$ for all $I \subset\{1, \ldots, n\}, \varepsilon= \pm 1$, and $k=1, \ldots, n-1$.

Lemma 2.6. Let $\left\{y_{1}, \ldots, y_{m}\right\}$ be a set of coset representatives for $Y_{I}$ in $B_{n}$. Let $J=\{1,2, \ldots, i\}$ and $I=\{i+1, i+2, \ldots, n\}$. If $\gamma \in B_{n}$ and $\alpha=$ $\alpha_{1} \alpha_{2} \in B_{n}^{i}$, where $\alpha_{1} \in\left\langle\sigma_{1}, \ldots, \sigma_{i-1}\right\rangle$ and $\alpha_{2} \in\left\langle\sigma_{i+1}, \ldots, \sigma_{n-1}\right\rangle$, then there is $k=1, \ldots, m$ such that $\varphi_{I}\left(y_{k} \gamma \alpha \gamma^{-1} y_{k}^{-1}\right)$ is a conjugate of $\alpha_{1}$ in $B_{n}$ and $\varphi_{J}\left(y_{k} \gamma \alpha \gamma^{-1} y_{k}^{-1}\right)$ is a conjugate of $\alpha_{2}$ in $B_{n}$.

Proof. For this choice of $\gamma$ there is $k=1, \ldots, m(i)$ such that $y_{k} \gamma$ is in $Y_{I}$. Then by Lemma 2.1 we see that

$$
\begin{aligned}
\varphi_{I}\left(y_{k} \gamma \alpha \gamma^{-1} y_{k}^{-1}\right) & =\varphi_{I}\left(y_{k} \gamma\right) \varphi_{I}\left(\alpha \gamma^{-1} y_{k}^{-1}\right) \\
& =\varphi_{I}\left(y_{k} \gamma\right) \varphi_{I}(\alpha) \varphi_{I}\left(\gamma^{-1} y_{k}^{-1}\right) \\
& =\varphi_{I}\left(y_{k} \gamma\right) \varphi_{I}\left(\alpha_{1} \alpha_{2}\right) \varphi_{I}\left(\gamma y_{k}\right)^{-1} \\
& =\varphi_{I}\left(y_{k} \gamma\right) \alpha_{1} \varphi_{I}\left(\gamma y_{k}\right)^{-1}
\end{aligned}
$$

is a conjugate of $\alpha_{1}$ in $B_{n}$ as required. A similar argument shows that $\varphi_{J}\left(y_{k} \gamma \alpha \gamma^{-1} y_{k}^{-1}\right)$ is a conjugate of $\varphi_{J}\left(\alpha_{2}\right)$; but one easily sees that $\varphi_{J}\left(\alpha_{2}\right)$ is a conjugate of $\alpha_{2}$ in $B_{n}$ and so the result follows.

For the moment let us keep the notation of Lemma 2.6. Now if $m \geq$ $i+j-1$, then we let $\Pi_{i}: B_{j} \rightarrow B_{m}$ be the monomorphism determined by $\Pi_{i}\left(\sigma_{k}\right)=\sigma_{k+i-1}$ for all $k=1, \ldots, j-1$. Then $\Pi_{i+1} \Sigma_{j}\left(\Sigma_{h}\right)=\Sigma_{h}$ for all $h=i+1, \ldots, n-1$. This fact, together with the proof of Lemma 2.6, gives the following result. 
Corollary 2.7. If $\alpha=\alpha_{1} \alpha_{2} \in B_{n}^{i}$, where $\alpha_{1} \in\left\langle\sigma_{1}, \ldots, \sigma_{i-1}\right\rangle$ and $\alpha_{2} \in$ $\left\langle\sigma_{i+1}, \ldots, \sigma_{n-1}\right\rangle$, then $\alpha$ is conjugate in $B_{n}$ to

$$
\left(\varphi_{I}\left(y_{k} \gamma \alpha \gamma^{-1} y_{k}^{-1}\right)\right)\left(\Pi_{i+1} \varphi_{J}\left(y_{k} \gamma \alpha \gamma^{-1} y_{k}^{-1}\right)\right) \text {. }
$$

We now describe the algorithm for determining if we can conjugate $\alpha \in B_{n}$ into some $B_{n}^{i}$. This is based on the above results and the fact that the conjugacy problem is solved in $B_{n}$.

Step 1 . For each $i=1,2, \ldots, n$ we let $I=\{1,2, \ldots, n\} \backslash\{i\}$ and find a set $\left\{y_{1}, \ldots, y_{m(i)}\right\}$ of coset representatives for $Y_{I}$ in $B_{n}$.

Do Steps $2(\mathrm{i})$ and $3(\mathrm{i})$ for each $i=1, \ldots, m(i)$. If $\alpha$ is conjugate to a braid in $B_{n}^{i}$, then we succeed for some value of $i$, by Corollary 2.7 .

Step 2(i). Let $w_{i}=\left(\varphi_{I}\left(y_{k} \gamma \alpha \gamma^{-1} y_{k}^{-1}\right)\right)\left(\Pi_{i+1} \varphi_{J}\left(y_{k} \gamma \alpha \gamma^{-1} y_{k}^{-1}\right)\right)$ where we calculate this braid using Proposition 2.3. The braid $w_{i}$ has a representation as a product of the standard generators and their inverses, which is calculated using an algorithm described in the proof of a theorem of Artin (see [Bi, p. 30]).

Step 3(i). Use the solution of the conjugacy problem as presented in [Ga] (see also [Bi]) to determine whether $w_{i}$ and $\alpha$ are conjugate in $B_{n}$.

If no $w_{i}$ is conjugate to $\alpha$, for all $k=1, \ldots, m(i)$, then $\alpha$ is not conjugate to a braid in $B_{n}^{i}$ by Corollary 2.7 .

\section{REFERENCES}

[Bi] J. Birman, Braids, links and mapping class groups, Ann. of Math. Studies, vol. 82, Princeton Univ. Press, Princeton, NJ, 1985.

[Ga] F. A. Garside, The Braid group and other groups, Quart. J. Math. Oxford Ser. (2) 20 (1969), 235-254.

[Ma] W. Magnus, Braid groups: a survey, Lecture Notes in Math., vol. 372, Springer-Verlag, New York, 1973, pp. 463-487.

Department of Mathematics, Brigham Young University, Provo, Utah 84602 